\title{
Política de gerenciamento, acesso e uso do Repositório Institucional do ILA (RILA)
}

\author{
Agenor de Oliveira Ikeda \\ Bacharel do Curso de Análise de Sistemas da Universidade Nove de Julho - São Paulo/SP \\ E-mail: ikedaaoi@fab.mil.br \\ Daniele Cristina lanni Rocha \\ Bacharel do Curso de Arquivologia da Universidade Estadual Paulista - Marília/SP \\ E-mail: iannidcir@fab.mil.br \\ Diego Antônio Fernandes Aguiar \\ Bacharel do Curso de Ciência da Computação da Universidade Federal de Tocantins - Tocantins/TO \\ E-mail: aguiardaafa@fab.mil.br \\ Marcela Cecília Inácio Evangelista \\ Bacharel do Curso de Biblioteconomia da Universidade Estadual Paulista - Marília/SP \\ E-mail: ceciliamcie@fab.mil.br \\ Marina Bortolotti Biudes \\ Bacharel do Curso de Biblioteconomia da Universidade Estadual Paulista - Marília/SP \\ E-mail: marinambb@fab.mil.br
}

\begin{abstract}
RESUMO:
O presente trabalho apresenta um relato sobre a Política de Gerenciamento, Acesso e Uso do Repositório Institucional do Instituto de Logística da Aeronáutica (RILA). Iniciando-se na fase de desenvolvimento da proposta e o estudo da implantação do projeto. A partir da Política do RILA, pretende-se estabelecer uma rotina de atividades e procedimentos a fim de, assegurar o recebimento, o tratamento, a preservação, o acesso e o uso dos documentos e/ou informações contidos no RILA. O RILA tem por finalidade organizar e preservar a documentação de caráter de ensino, técnico-científico, técnico-administrativo e de memória produzidas no âmbito do Instituto de Logística da Aeronáutica (ILA), visando a qualidade no gerenciamento da informação e da documentação institucional. Objetiva-se prover visibilidade e acesso pontual às produções bibliográficas e documentais produzidas no Instituto, em atendimento ao que preveem as legislações vigentes de acesso à informação.
\end{abstract}

Palavras-chave: Repositórios digitais. Conservação de documentos. Políticas de informação. Gestão do conhecimento.

\footnotetext{
ABSTRACT:

The present work presents hum account About Management Policy, Access and Use of Institutional Repository Logistics Institute of Aeronautics (RILA). Starting on the development phase of the Proposal and the Study of Project Implementation. At from the RILA Policy, we intend to establish a routine activities and procedures an End to ensure receipt, treatment, Preservation, Access and use of documents and / or information contained in RILA. The RILA is intended to establish and preserve the educational character of documentation, technical-scientific, technical, administrative and memory produced in the Scope of Logistics Institute of Aeronautics (ILA), to the Quality of Information Management and institutional documentation. It aims to provide visibility and timely access to bibliographic and documentary productions produced in the Institute, in compliance with the que predict how current legislation on Access to Information.
} 
Keywords: Digital Repositories. Materials conservation. Information policy. Knowledge management.

\section{INTRODUÇÃO}

O Instituto de Logística da Aeronáutica (ILA), criado pelo Decreto $n^{\circ}$ 95.640, de 13 de janeiro de 1988, é uma organização militar do Comando da Aeronáutica (COMAER) cuja missão é desenvolver as capacidades técnicas e gerenciais dos profissionais do COMAER por intermédio das atividades de ensino e pesquisa, em proveito dos sistemas logísticos, conforme previsto em seu Regimento Interno (RICA 21-50/2011), publicado pela Portaria COMGAP n²8/3EM, de 21 de julho de 2011.

$\mathrm{Na}$ área de ensino, o ILA promove a atualização técnica e a capacitação de recursos humanos para o exercício de funções específicas na área da logística aeronáutica, sob a égide do Comando-Geral de Apoio (COMGAP), conforme prevê o Parágrafo $1^{\circ}$ do Art. 16 do Decreto $\mathrm{n}^{\circ}$ 6.834, de 30 de abril de 2009, que dispõe sobre a subordinação do ILA ao COMGAP. Isto se dá por meio de ampla gama de cursos presenciais, semipresenciais e à distância, elaborados para o aprimoramento constante do capital humano afeto à área da logística militar.

$\mathrm{Na}$ área de pesquisa, o ILA desenvolve atividades de investigação científica, por meio das quais tem assessorado diversas organizações do COMAER na solução de problemas logísticos e na otimização dos seus processos produtivos.

O ILA possui um acervo multidisciplinar voltado à área da logística do COMAER, composto por um rol de documentos/itens informacionais que tramitam no Instituto, e que não possuem acesso digital tratado e facilitado. Esses documentos são das áreas de: Ensino - Relatórios de Coordenação de Cursos e de Grupos de Trabalho (GT), Plano de Unidades Didáticas (PUD), Currículos Mínimos (tanto os que vigoram quanto os desatualizados), Quadros-Horários (QH) dos cursos, Questionários Didático-Pedagógicos para os GT, Materiais Didáticos (textuais e midiáticos), Videoaulas, Bancos de Imagens e Filmagens relacionadas aos eventos e cursos transcorridos interna e externamente ao ILA, entre outros; Apoio Administrativo - Guias de Recolhimento de Receitas, Relatórios e Controles de 
Despesas, Termos de Referência, Projetos Básicos, Pareceres Técnicos, entre outros; Pesquisa - Relatório de Consultorias Logísticas, Projetos de Capacitação, Levantamentos e Apresentações Digitais Setoriais e Institucionais, Teses e Dissertações dos alunos dos cursos de Especialização Lato Sensu em Logística e em Tecnologia da Informação, e dos alunos dos cursos de Mestrado Stricto Sensu na Air Force Institute of Technology (AFIT) e na Cranfield University, outras documentações afetas às Divisões e aos Setores producentes do ILA.

Estas documentações, por sua importância no âmbito interno e externo ao ILA e ao COMAER, necessitam ser organizadas e dispostas de tal forma que sua recuperação e seu acesso sejam ágeis e fáceis, para a manutenibilidade da memória histórica da Instituição e, consequentemente, para a reafirmação da identidade organizacional, visibilidade e acesso à produção intelectual do ILA. Isto corrobora com o disposto nas legislações que se seguem: Art. $5^{\circ}$ da Lei $n^{\circ} 12.527$ (Lei de Acesso à Informação, LAI), de 18 de novembro de 2011, que diz que "é dever do Estado garantir o direito de acesso à informação, que será franqueada, mediante procedimentos objetivos e ágeis, de forma transparente, clara e em linguagem de fácil compreensão."; e com o Art. $2^{\circ}$ do Decreto Regulamentador da LAI n 7. 724, de 16 de maio de 2012, que diz que:

Os órgãos e as entidades do Poder Executivo federal assegurarão, às
pessoas naturais e jurídicas, o direito de acesso à informação, que será
proporcionado mediante procedimentos objetivos e ágeis, de forma
transparente, clara e em linguagem de fácil compreensão, observados os
princípios da administração pública e as diretrizes previstas na Lei no
12.527, de 2011 .

Com a Constituição Federal Brasileira, em seu Inciso XXXIII, Capítulo I, que dispõe que:

Todos têm direito a receber dos órgãos públicos informações de seu interesse particular, ou de interesse coletivo ou geral, que serão prestadas no prazo da lei, sob pena de responsabilidade, ressalvadas aquelas cujo sigilo seja imprescindível à segurança da sociedade e do Estado.

E em seu Art. $216^{\circ}$, Parágrafo $2^{\circ}$, onde diz que "cabe à administração pública, na forma da lei, a gestão da documentação governamental e as providências para franquear sua consulta a quantos dela necessitem".

Neste contexto, há um software livre, denominado DSpace que foi 
desenvolvido pelo Massachusetts Institute of Technology (MIT) e pelos Laboratórios Hewlett-Packard (HP) com o fito de criar repositórios institucionais e multidisciplinares para bibliotecas, arquivos e centros de pesquisa. Este software é tutelado pelo Instituto Brasileiro de Informação em Ciência e Tecnologia (IBICT) e é um dos mais utilizados no Brasil, por dezenas de entidades públicas e privadas, entre elas a Universidade de São Paulo (USP), Universidade Federal do Rio Grande do Sul (UFRGS), Fundação Oswaldo Cruz (FIOCRUZ), Superior Tribunal de Justiça (STJ), Senado Federal, entre outras, conforme informações do Ministério da Ciência, Tecnologia e Inovação (MCTI).

Segundo Leite (2007), o ambiente acadêmico, possui uma estrutura cultural, social e tecnológica constituída de forma favorável às práticas da gestão do conhecimento. Obtém-se o uso de repositórios como uma ferramenta de métodos de arquivamento ideal para a preservação da memória deste Instituto.

Os repositórios institucionais são bases de dados online que reúnem de maneira organizada a produção científica/intelectual de uma instituição. Eles armazenam arquivos de diversos formatos (ppt, pdf, txt, jpeg, mpeg, tiff, etc.), possuem interoperabilidade com sistemas congêneres por meio do protocolo $\mathrm{OAI} / \mathrm{PMH}$, podem também ser customizados de acordo com as características da Instituição, dentre diversas outras facilidades, que resultam, segundo o IBICT 2016 em diversos benefícios para pesquisadores de instituições, proporcionando maior visibilidade aos resultados de pesquisas e possibilitando a preservação da história científica da instituição.

\section{DESENVOLVIMENTO}

Para a proposta de implementação do Repositório, foi feito um estudo e análises de Repositórios já implantados em outras instituições, como por exemplo, da Escola Nacional de Administração Pública (ENAP). Para Costa e Leite (2009) os Repositórios possuem atributos importantes, das quais procurou-se abranger por meio da Política do RILA, quais sejam:

- Ser institucionalmente definido: seus limites são definidos pelas fronteiras da instituição, devem ser oficialmente reconhecidos pela instituição por meio de implementação de políticas que garantam sua existência;

- Orientação científica e acadêmica: cientificamente orientado deve significar conteúdo reconhecido e validado pela comunidade científica. 
Academicamente orientado flexibiliza a perspectiva da validação alcançada pelo peer review, e contemplam também outras formas de comunicação científica, mais informal, inclusive materiais de ensino;

- Cumulativo e perpétuo: preservar a produção intelectual de uma instituição e garantir acesso amplo e irrestrito; e

- Aberto e interoperável: uma das principais características responsáveis pelo aumento do impacto dos resultados de pesquisa e visibilidade da produção, do pesquisador e da instituição.

A partir das informações acima, pensando no total entendimento acerca do funcionamento do RILA, procurou-se definir em sua Política de Gerenciamento, Acesso e Uso, primeiramente os operadores do Repositório, seguido pela Organização do RILA, a partir da definição de Comunidades e Subcomunidades e do processo de submissão, a seguir abordou-se o acesso aos documentos dispostos no RILA, uso dos documentos e a questão dos Direitos Autorais.

\section{OPERADORES DO RILA}

A seguir estão definidos os responsáveis e operadores pelos processos do RILA:

- Usuários: Servidores militares ou civis dos efetivos do ILA e das demais Organizações Militares do COMAER que fazem uso dos produtos e serviços oferecidos pelo ILA;

- Autores: Setores do ILA que produzam documentação relevante para armazenamento permanente, bem como os militares/civis que desenvolvam conteúdo intelectual vinculado ao Instituto e/ou que estejam no exercício de suas funções;

- Submetedores (Depositantes): Encarregados de cada setor que produza documentação relevante para armazenamento permanente. Estes militares são os responsáveis pela submissão de itens informacionais de seu setor ao RILA, respeitando os critérios da Política. Eles fazem a descrição do item por meio dos metadados estabelecidos e upload do arquivo digital;

- Comissão de Avaliação e Revisão: Comissão publicada em Boletim Interno, composta por 02 Oficiais (arquivista, bibliotecário, ou outros profissionais qualificados na área de Gestão da Informação) e por 08 
Graduados. A Comissão é responsável pelo papel de Avaliador -faz a análise inicial e aprova o conteúdo submetido pelos Submetedores, indicando que o item informacional poderá ou não ser depositado no RILA, verificando restrições/autorizações relacionadas aos Direitos Autorais e permissões de uso - e de Revisor - responsável pela revisão final e publicação dos conteúdos submetidos e aprovados;

- Gerenciadores: A Seção de Arquivo Geral do ILA (AARQ) e a Biblioteca do ILA (PDBI) são responsáveis pela melhoria e evolução do RILA, atualização da política, concessão de perfis de acesso ao Repositório, atualização das informações do sistema, gestão das comunidades e coleções;

- Administradores: A Seção de Tecnologia da Informação do ILA (ASTI)é responsável pela gestão e manutenção do RILA, no que tange à infraestrutura tecnológica, atualização do software, configuração do sistema, layout do portal; e

- Comitê Gestor: Instituído caso haja necessidade de avaliar a pertinência de um novo item informacional e/ou objeto digital a ser armazenado no RILA. Constitui esse Comitê o Sr. Diretor do ILA, o Chefe do Setor que produz o conteúdo e os Gerenciadores e Administradores do RILA; e

- Biblioteca do ILA: A PDBI é a responsável pela recepção e gerenciamento da documentação relativa aos direitos autorais dos itens informacionais a serem submetidos ao RILA, bem como ao cadastro e controle de usuários que requererem acesso aos documentos de caráter restrito.

Após definição dos responsáveis, abordou-se a Organização do Repositório, conforme será abordado no tópico seguinte.

\section{ORGANIZAÇÃO DO RILA}

O RILA é organizado hierarquicamente em Comunidade e Subcomunidades, que, por sua vez, apresentam seus conteúdos dispostos em Coleções. As Comunidades representam o maior nível hierárquico na organização da informação no Repositório. As Subcomunidades são criadas dentro de uma Comunidade e servem para ajudar na organização do Repositório, com a possibilidade de adicionar Rev. Inf. na Soc. Contemp., Natal, RN, Número Especial, 2017| 
novos níveis hierárquicos. As Coleções são os conjuntos de itens informacionais, na qual os documentos são armazenados virtualmente. São subordinadas às Comunidades ou Subcomunidades.

Exemplo:

Repositório Institucional (Comunidade)

Históricos de criação (Subcomunidade)

Apresentações Institucionais do RILA (Coleções)

Políticas Institucionais do RILA (Coleções)

\subsection{INCLUSÃO DE COMUNIDADES, SUBCOMUNIDADES E COLEÇÕES}

A inclusão de novas Comunidades, Subcomunidades e Coleções, deve estar alinhada com os objetivos do RILA e estar sujeita a parecer favorável do Comitê Gestor.

O Comitê Gestor deverá avaliar a inclusão mediante os seguintes aspectos:

1. Conteúdo: avaliar o conteúdo de forma a assegurar a qualidade e adequação do mesmo aos objetivos do RILA;

2. Formato: avaliar o formato dos conteúdos digitaisde forma a garantir o acesso ao conteúdo dos arquivos e manter a padronização do RILA;

3. Participação do solicitante: garantir a participação do proponente no processo de definição e registro dos metadados, e da delimitação do fluxo de submissão (Submetedor) da nova Comunidade / Subcomunidade / Coleção.

A próxima fase a ser abordada na Política do RILA, trata-se da submissão, conforme abordou-se no próximo tópico.

\section{SUBMISSÃO}

Os documentos a serem submetidos ao RILA devem atender aos seguintes 
critérios:

- Estarem em formato digital, conforme definido pelos Administradores e Gerenciadores;

- Terem sido produzidos ou patrocinados pelo ILA e/ou COMAER;

- Estarem completos e finalizados, ou seja, não pertencerem ao arquivo corrente, e assinados, se for o caso;

- Estarem classificados como Ostensivos;

- Não serem passíveis de proteção de Propriedade Intelectual ou Industrial; e

- Não possuírem restrições contratuais relativas aos Direitos Autorais.

- Quanto aos metadados utilizados para submissão de documentos no RILA devem seguir o padrão Dublin Core e são definidos pelos Gerenciadores do Repositório, em conjunto com os Autores.

\section{ACESSO AOS DOCUMENTOS}

O acesso aos documentos depositados no RILA ocorre da seguinte forma:

- Acesso Aberto: os documentos são disponibilizados sem restrição de acesso, ou seja, sem a necessidade de login e senha;

- Acesso Restrito: os documentos têm seu acesso condicionado à identificação por login e senha;

- Acesso Embargado: os documentos têm seu acesso embargado por tempo determinado, ou seja, os documentos ficam indisponíveis para acesso por um período de tempo, e após findado este período, os documentos poderão ter seu acesso restrito ou aberto, conforme previamente configurado; e

- Acesso Fechado:os documentos possuem acesso limitado aos Gerenciadores e Administradores. Utilizado somente para cômputo de dados estatísticos, de gestão ou de memória institucional.

A solicitação de login e senha deverá ser feita junto à Biblioteca do ILA, Rev. Inf. na Soc. Contemp., Natal, RN, Número Especial, 2017| 
mediante preenchimento de um formulário e de um termo de ciência e responsabilidade.

\section{USO DOS DOCUMENTOS}

O uso dos documentos depositados no RILA será da seguinte forma:

- Os documentos depositados no RILA estão disponíveis sem ônus ao usuário, para fins de consulta e pesquisa, de acordo com as licenças adotadas para cada documento, conforme Política de Direitos Autorais do RILA;

- É vedada a reprodução, divulgação, comercialização e alteração dos documentos depositados no RILA sem a prévia autorização do autor;

- A divulgação e a reprodução, devidamente autorizada pelos autores, das informações contidas nos documentos no âmbito externo ao COMAER, deverá ser analisada e também autorizada pelo Comitê Gestor;

- Os usuários que utilizarem os recursos disponíveis no RILA deverão citar o nome do autor e os dados completos da obra; e

- É vedado o uso da documentação disponível no RILA em desconformidade com a Lei de Direitos Autorais Brasileira, vigente.

\section{DIREITOS AUTORAIS}

Conforme disposto nos Incisos do Art.7 da Lei de Direitos Autorais $\left(\mathrm{n}^{\circ}\right.$ 9.610/98), as obras intelectuais armazenadas no RILA são protegidas, portanto, conforme Inciso I do Art. 29 da citada Lei, faz-se necessária a autorização prévia e expressa do autor das obras inseridas no RILA, nos casos em que o autor não possua vínculo institucional com ILA, conforme estabelecido na Política de Direitos Autorais do ILA. Para isso, consta no foi feita uma Declaração e um Termo de Autorização que deverão ser preenchidos pelo autor de cada obra a ser depositada no RILA, anteriormente ao seu depósito. Esta Declaração deverá ser preenchida, assinada e entregue na Biblioteca do ILA.

As demais disposições relacionadas às licenças de uso, domínio público e 
autorizações constam na Política de Direitos Autorais do RILA, devendo esta ser verificada pelos usuários do RILA, obrigatoriamente.

\section{CONSIDERAÇÕES FINAIS}

A estrutura das Comunidades, Subcomunidades e Coleções, a tipologia, os procedimentos de submissão dos itens informacionais, as normas de preenchimento dos conjuntos de metadados, as etapas do fluxo de submissão serão descritos no Manual do RILA, desenvolvido com o intuito de colaborar com a compreensão e utilização dessa ferramenta

Tendo em vista a necessidade de um armazenamento digital permanente para a salvaguarda dos documentos anteriormente citados, com vistas à preservação das documentações produzidas no âmbito do Instituto, ao atendimento às legislações vigentes, e, levando em consideração a necessidade de disponibilidade e acesso à produção documental de ensino, pesquisa e apoio do ILA, faz-se premente a criação e implementação de um repositório digital institucional no ILA. Para tanto, originou-se um projeto, tendo como objetivo geral possibilitar a implementação do Repositório Institucional do Instituto de Logística da Aeronáutica (RILA), e, como objetivo específico, permitir a gerência das ações e prazos para a efetiva implantação e gerência do RILA. O projeto supracitado esta em fase de implantação.

\section{REFERÊNCIAS}

BRASIL. Decreto Lei no 6.834 , de 30 de abril de 2009. Aprova a Estrutura Regimental e o Quadro Demonstrativo dos Cargos em Comissão do Grupo-Direção e Assessoramento Superiores e das Funções Gratificadas do Comando da Aeronáutica, do Ministério da Defesa, e dá outras providências. Diário Oficial [da] Republica Federativa do Brasil, Brasília, DF, 30 abr. 2009.

. Decreto Lei $\mathrm{n}$ - 7.724, de 16 de maio de 2012. Regulamenta a Lei $\mathrm{n} 012.527$, de 18 de novembro de 2011, que dispõe sobre o acesso a informações previsto no inciso XXXIII do caput do art. 5ำ, no inciso II do $\S 3^{\circ}$ do art. 37 e no $\S 2^{\circ}$ do art. 216 
da Constituição. Diário Oficial [da] Republica Federativa do Brasil, Brasília, DF, 16 mai. 2012.

BRASIL. Lei no 12.527, de 18 de novembro de 2011. Regula o acesso a informações previsto no inciso XXXIII do art. 5o, no inciso II do § 30 do art. 37 e no § 20 do art. 216 da Constituição Federal; altera a Lei no 8.112, de 11 de dezembro de 1990; revoga a Lei no 11.111, de 5 de maio de 2005, e dispositivos da Lei no 8.159 , de 8 de janeiro de 1991; e dá outras providências. Diário Oficial [da] Republica Federativa do Brasil, Brasília, DF, 18 novembro 2011.

BRASIL. Constituição (1988). Constituição da República Federativa do Brasil. Brasília, DF: Senado Federal: Centro Gráfico, 1988. 292 p.

BRASIL. Ministério da Ciência, Tecnologia e Inovação. Informações dobre o

Software DSpace. Disponível em: < http://www.mcti.gov.br/> Acesso em: 16 de junho de 2016.

BRASIL. Ministério da Defesa. Comando da Aeronáutica. Comando-Geral do Pessoal. Confecção, Controle e numeração de publicações Oficiais do Comando da Aeronáutica - NSCA 5-1. [Rio de Janeiro]: [S.I], 23 nov. 2011. 67 p.

Correspondência e Atos Oficiais do Comando da Aeronáutica (ICAER) - ICA 10-1. [Brasília]: [S.I], 12 fev. 2015. 149 p.

BRASIL. Ministério da Defesa. Comando da Aeronáutica. Instituto de Logística da Aeronáutica. Modelo e Norma para Confecção, Controle e Numeração das NPA do ILA - NPA 5-1. São Paulo, 16 jul. 2015.

BRASIL. Ministério da Defesa. Comando da Aeronáutica. Instituto de Logística da Aeronáutica. Regimento Interno do Instituto de Logística da Aeronáutica - RICA 2150/2011. São Paulo, 21 jul. 2011.

ESCOLA NACIONAL DE ADMINISTRAÇÃO PÚBLICA. Política do repositório institucional da ENAP. Brasília, [2013?]. Disponível em:

<repositório.enap.gov.br/politica>. Acesso em: 25 maio 2016.

ESCOLA NACIONAL DE ADMINISTRAÇÃO PÚBLICA. Diretoria de Desenvolvimento Gerencial Coordenação Geral de Educação a Distância. Gerência de Projetos: teoria e prática: módulo 5: elaboração de uma EAP. Brasília, 2014. Disponível em: <repositório.enap.gov.br/politica>. Acesso em: 15 jun. 2016.

INSTITUTO BRASILEIRO DE INFORMAÇÃO E TECNOLOGIA. Repositório Institucional do Instituto Brasileiro de Informação em Ciência e Tecnologia. Disponível em: < http://repositorio.ibict.br/> Acesso em: 16 de junho de 2016.

LEITE, F. C.L.; COSTA, S. Repositórios institucionais como ferramentas de gestão do conhecimento cientifíco no ambiente acadêmico. Persp. Ciênc. Inf., Belo Horizonte, v. 11, n. 2, p. 206-219, maio/ago. 2006.

RIBEIRO JUNIOR, Divino Ignácio [et. al.. Implantação do repositório digital do 
projeto 'memória científica da Faed' com dspace: relato de experiência. Revista ACB: Biblioteconomia, Florianópolis, v.17, n.1, p. 152-173, jan./jun. 2012. Disponível em: << https://revista.acbsc.org.br/racb/article/view/824>>. Acesso em: 14 junho 2016.

SANTOS, Henrique Machado; FLORE, Daniel. Políticas de preservação digital para documentos arquivísticos. Perspectivas em Ciência da Informação. v. 20, n. 4, p.197217, out./dez. 2015. Disponível em :<http://www.scielo.br/pdf/pci/v20n4/1413-9936pci-20-04-00197.pdf>. Acesso em: 14 jun. 2016.

SAYÃO, Luis; TOUTAIN, Lídia Brandão; ROSA, Flavia Garcia; MARCONDES, Carlos Henrique (Org.). Implantação e gestão de repositórios institucionais: políticas, memória, livre acesso e preservação. Salvador: Edufba, 2009.

UNIVERSIDADE DE SÃO PAULO. Biblioteca digital da produção intelectual: resolução no 6.444, de 22 de outubro de 2012. Reitoria da USP: São Paulo, 2012. Disponível em: <www.producao.usp.br/page/politicaAcessoPtBR>. Acesso em: 25 maio 2016.

UNIVERSIDADE FEDERAL DA INTEGRAÇÃO LATINO-AMERICANA.. Biblioteca Latino-americana. Política de informação do repositório institucional da UNILA. UNILA: Foz do Iguaçu, 2015. Disponível em: <www.unila.edu.br/sites/default/files/ files/Politica RI-UNILA_Nov_2015.pdf>. Acesso em: 30 maio 2016.

UNIVERSIDADE FEDERAL DO RIO GRANDE DO SUL. Política institucional de informação para o LUME: repositório digital da Universidade Federal do Rio Grande do Sul - Portaria no 5068, de 13 de outubro de 2010. [Porto Alegre]: Reitoria da UFRGS, 2010. Disponível em: <www.lume.ufrgs.br/arquivos_download/Portaria5068.pdf>. Acesso em: 25 maio 2016.

VIANNA, Sheila Maria de Vasconcellos; CARVALHO, Rogerio Atem de. Benefícios da implantação de repositório institucional na preservação da memória institucional. Florianópolis, 2013. Disponível em: <https://portal.febab.org.br/ anais/article/view/1613 >. Acesso em: 14 jun. 2016.

WEITZEL, Simone da Rocha; MACHADO, Elisa Campos. Estratégias para implementação de repositórios da UNIRIO. In: SEMINÁRIO NACIONAL DE BIBLIOTECAS UNIVERSITÁRIAS, 16., 2010, Rio de Janeiro. [Conference paper]. Disponível em: <http://eprints.rclis.org/15732/>. Acesso em: 14 jun. 2016. 